\title{
Morphofunctional characteristics of mouse (Mus musculus musculus) liver on the application of various doses of nanostructural sapropel
}

\author{
Ramil N. Faizrakhmanov ${ }^{1}$, Yuliya V. Larina ${ }^{1,2, *}$, Asiya M. Ezhkova ${ }^{2}$, Vladimir O. Ezhkov ${ }^{2}$, and Ekaterina Semakina ${ }^{2}$ \\ ${ }^{1}$ Kazan State Academy of Veterinary Medicine named after N.E. Bauman, 420029 Kazan, Russia \\ ${ }^{2}$ Tatar Research Institute of Agricultural Chemistry and Soil Science - Subdivision of the Federal Research Center "Kazan Scientific \\ Center of Russian Academy of Sciences", 420059 Kazan, Russia
}

\begin{abstract}
The liver is considered to be the main organ in the processes of regulating metabolism, neutralizing toxins and maintaining the constancy of the internal environment of the body. The goal of the research was to study the morphofunctional state of the liver under the effect of different concentrations of nanostructured sapropel. The experiments were carried out on non-linear (outbred) white mice weighing $24.9 \pm 1.8 \mathrm{~g}$. Twelve mature males were allotted to four groups. Mice of the experimental groups I, II and III intragastrically through the atraumatic flexible probe were once injected with nanostructured sapropel (particle size of $45.0-180.0 \mathrm{~nm}$ ) in the following doses: lethal $-3.0 \mathrm{~g} / \mathrm{kg}$ of the body weight; toxic $1.8 \mathrm{~g} / \mathrm{kg}$ of the body weight and safe $-0.6 \mathrm{~g} / \mathrm{kg}$ of the body weight. Mice of group IV served as a control one and received deionized water in the same way. The choice of liver as the organ for analyzing is justified by the fact that the liver did not have direct contact with sapropel nanoparticles in the process of its intragastric administration into the body of white mice. Four hours after the introduction of nanostructured sapropel, three mice from each group were killed by cervical dislocation. After preparation and staining with hematoxylin and eosin, identical pieces of the liver were evaluated using light microscopy. Histological studies have established that the introduction of a lethal dose of nanostructured sapropel caused hemodynamic vascular disorders; focal necrosis and necrobiosis of hepatocytes were also observed in the research. Furthermore, the research noted a migration of reticuloendotheliocytes to the centrolobular regions of the lobules and enhancement of their activity. The microstructure of the liver when introducing a toxic dose of nanostructured sapropel was characterized by moderate plethora of sinusoidal capillaries, deformation of hepatocytes, focal destruction with the development of karyopiknosis, karyorhexis and karyolysis. The study revealed the activation of reticuloendothelial cells. Liver histology when introducing a safe dose of nanostructured sapropel was characterized by the preservation of the integrity of the structural elements, polyploid (two- and multi-core) hepatocytes were identified in the periportal part of the lobes. The changes in the structural and functional state of the mice liver were found to be depending on the dose of the nanostructured sapropel.
\end{abstract}

\section{Introduction}

The liver (hepar, liver) is the largest digestive gland of a complex tubular structure in mammals. It has a redbrown color, an elastic consistency, a flattened convexconcave shape and a lobular structure. The main functional-morphological unit of the organ is the hepatic lobules, which, merging together, form a complex lobule. Liver lobules have various forms: oval, polyhedral, in the form of a multilateral prism wherein the central vein passes. From the central vein, the hepatic tubules radially extend, consisting of double-row hepatic cells of various sizes, having one or two nucleus. In addition to hepatocytes, connective tissue elements such as fibroblasts and sinusoidal cells play an important role in the structure of the organ: endothelial cells, star reticuloendothelial cells (Kupffer's cells), perisinusoid cells (lipocytes, Ito cells, vitamin A synthesized, granulosa (pit-cells) $[5,10,16]$.

Numerous and important functions of the liver determine its importance as a vital organ. The liver is the main organ in the regulation of metabolism and maintaining the uniformity of the internal environment of the organism. The liver is one of the important target organs that respond quickly to the effects of exogenous and endogenous stimuli $[3,18]$.

To our knowledge, in recent years, drugs with high level nanoparticles have been used in animal husbandry $[8,9,14]$. The application of nanoparticles leaves open the question of their effects on living organisms [2]. The authors' works show the potential danger of the destructive effects of nanoparticles on the mucous

Corresponding author: dskgavm@mail.ru 
membranes of the digestive and respiratory systems $[6,21,23]$. The article by Jo D.H. shows the mechanism of nanoparticle penetration into the central nervous system along the nerve fibers from the olfactory epithelium of the retina [11].

However, some researchers have reported that nanoparticles do not have a toxic effect. Nanoparticles of a water solution of $\mathrm{CdSe} / \mathrm{ZnS}$, stabilized by cysteine, do not have a cytotoxic effect when they are absorbed by cells [19].

Researchers believe that the manifestation of the negative properties of nanoparticles in living organisms is significantly influenced by the shape and small size. The nanoparticles of the dendric and spindle-shaped forms have comparatively greater cytotoxicity and cause more permissive effects in the body than spherical particles $[12,17,24]$. Tsoli, M. [20] showed the dependence of toxicity on particle size: large gold particles had no toxic properties, while gold nanoparticles with a size of $1.4 \mathrm{~nm}$ had a destructive effect on cellular structures. Some researchers [13, 15, 22] believe that the dose of the drug has a significant effect when exposed to nanoparticles on the body.

There is evidence of feed additives production of a new generation based on natural nanostructured minerals. They actively influence the biology of a living organism as compared to native analogues [1, 4]. Sapropel is a unique natural organic-mineral complex, produced from centuries-old bottom deposits of freshwater reservoirs. It is composed of low-molecular organic compounds, vitamins, carotenoids, enzymes and microelements in bioavailable form. The use of sapropel in feeding of farm animals increases their efficiency and improves the quality of products [7].

The present research therefore was aimed at studying the morphofunctional state of the liver under the influence of nanostructural sapropel. The research was focused more on the morphology of the liver, as the lastmentioned organ had no direct contact with sapropel nanoparticles when intragastrically introduced into the organism. In contrast, the liver, as the main target organ, actively responded to the various doses of nanostructural sapropel.

\section{Materials and methods}

This research was focused on the non-pedigreed white mice (Mus musculus musculus), their liver and nanosructural sapropel with particles of 45.0-180.0 nm.

For the manufacture of nanostructured sapropel, the researchers used sapropel (Beloye Ozero (White Lake), Republic of Tatarstan, Russian Federation) dried up to $1.0 \%$ humidity and mechanically crushed to $0.18 \mathrm{~mm}$. The elemental composition of the sapropel of this deposit is represented by a wide range of biogenic macro-and microelements (Table 1).

Nanostructured sapropel was processed via ultrasonic treatment in Kazan Research and Innovative-Application Centre "Nanomaterials and Nanotechnologies". Sapropel was added to deionized water at a concentration of $10 \mathrm{~g}$ per $100 \mathrm{ml}$ of water. Then the resulting suspension was subjected to ultrasonic treatment in an ultrasonic device0.25 at a frequency of $18.5 \mathrm{kHz}( \pm 10 \%)$, specific power of $80 \mathrm{~W} / \mathrm{l}$, amplitude of oscillation of the ultrasonic waveguide of $5 \mu \mathrm{m}$. The duration of the dispersion was 30 minutes. The size and shape of the particles of native sapropel and nanostructured sapropel were studied using by a MultiMode $\mathrm{V}$ scanning probe microscope (Veeco, USA) in the center mentioned above.

Table 1. Elemental composition of sapropel, $\mathrm{mg} / \mathrm{l}$

\begin{tabular}{|c|c|c|}
\hline № & Element & Content \\
\hline 1 & $\mathrm{Ca}$ & 2.40 \\
\hline 2 & $\mathrm{Fe}$ & 0.68 \\
\hline 3 & $\mathrm{Al}$ & 0.390 \\
\hline 4 & $\mathrm{Mg}$ & 0.350 \\
\hline 5 & $\mathrm{Sr}$ & 0.077 \\
\hline 6 & $\mathrm{~K}$ & 0.048 \\
\hline 7 & $\mathrm{P}$ & 0.047 \\
\hline 8 & $\mathrm{Mn}$ & 0.046 \\
\hline 9 & $\mathrm{Na}$ & 0.020 \\
\hline 10 & $\mathrm{Si}$ & 0.018 \\
\hline 11 & $\mathrm{Ti}$ & 0.011 \\
\hline 12 & $\mathrm{Ba}$ & 0.008 \\
\hline 13 & $\mathrm{Zn}$ & 0.0023 \\
\hline 14 & $\mathrm{Ni}$ & 0.0023 \\
\hline 15 & $\mathrm{~V}$ & 0.002 \\
\hline 16 & $\mathrm{Li}$ & 0.001 \\
\hline 17 & $\mathrm{Cr}$ & 0.0015 \\
\hline 18 & $\mathrm{Cu}$ & 0.0014 \\
\hline 19 & $\mathrm{Co}$ & 0.0005 \\
\hline 20 & $\mathrm{~Pb}$ & 0.0005 \\
\hline
\end{tabular}

The experiments were carried out on non-pedigreed white male mice (taken from the vivarium of the Tatar Interregional Veterinary Laboratory, Kazan) at 4 months of age and $24.9 \pm 1.8 \mathrm{~g}$ of the body weight. Mice were previously placed under quarantine within 12 days, and immediately before the experiment were kept without water and feed for 8 hours.

To determine the acute oral toxicity of a nanostructured sapropel, the mice were allotted to four experimental groups. Mice of the experimental groups I, II and III intragastrically through the atraumatic flexible probe were once injected with nanostructured sapropel at the following doses: lethal $-3.0 \mathrm{~g} / \mathrm{kg}$ of the body weight; toxic $-1.8 \mathrm{~g} / \mathrm{kg}$ of the body weight and safe $-0.6 \mathrm{~g} / \mathrm{kg}$ of the body weight. The mice of the group IV served as a control one and received deionized water in the same way (Table 2).

Table 2. The number of injected substances and the death of mice

\begin{tabular}{|c|c|c|c|c|}
\hline $\begin{array}{c}\text { Group } \\
(\mathrm{n}=12)\end{array}$ & $\begin{array}{c}\text { Dose, } \\
\mathrm{g} / \mathrm{kg}\end{array}$ & $\begin{array}{c}\text { Amount, } \\
\mathrm{g} / \text { animal }\end{array}$ & $\begin{array}{c}\text { Amount of } \\
\text { water, } \\
\mathrm{cm}^{3} / \text { animal }\end{array}$ & $\begin{array}{c}\text { Death of } \\
\text { mice, } \\
\text { animal }\end{array}$ \\
\hline I & 3.0 & 0.075 & 0.5 & 1 \\
\hline II & 1.8 & 0.045 & 0.5 & 0 \\
\hline III & 0.6 & 0.015 & 0.5 & 0 \\
\hline IV & - & - & 0.5 & 0 \\
\hline
\end{tabular}

Nanostructured sapropel in the form of an aqueous suspension and deionized water were introduced into the body of mice using an atraumatic probe. For histological 
observations, four hours after the introduction of nanostructured sapropel, three mice from each group were killed by cervical dislocation.

Autopsy of white mice was performed by evisceration according to G.V. Shor. For histological studies, segments of the liver were fixed in a $10 \%$ water solution of formalin, followed by condensation in the freezing microtomes with cooling "OMT-0228" and "MZP-01 Tehnom". Histosections were stained with Bemer's hematoxylin and $0.1 \%$ water solution of eosin. Histological preparations were analyzed using light microscopy (MBI-1) with magnification of a ring $(\times 7$, $\times 10$, and $\times 15)$ of lens $(\times 10, \times 20$, and $\times 40)$. The "JENAMED2" microscope with a ring GF-PW 10x25 of lens 40 was used for photographing of microscopic specimens.

All experiments were carried out in accordance with "the International Recommendations for conducting biomedical research using animals" (1985) and Order No. 708n of the Ministry of Health and Social Development of the Russian Federation dated August 23, 2010, "On Approval of Laboratory Practice Rules".

All procedures in the present work were approved by the Ethical Committee for Biological Research using experimental animals at the Federal Center for Toxicological, Radiation and Biological Safety, Kazan, Russia (Protocol №2 of 22 January 2019).

Statistical processing of digital experimental material was carried out in Microsoft Excel; the Student's tcriteria was implemented to determine the significance of the differences. To test the normality of distribution the method of moments was used, and the homogeneity of the dispersions was analyzed with the Fisher criteria.

\section{Results}

In the autopsy of mice that received a lethal dose of nanostructural sapropel, the macroscopic image of the digestive organs was characterized by hemodynamic vascular disorders: hyperemia, banded hemorrhages of the gastric mucosa and petechial hemorrhages of the intestinal mucosa. In the lumen of the digestive tube, more intensely in the intestinal section, clumps of sapropel were identified. Parenchymal organs (kidneys, spleen, liver) were slightly enlarged in volume, had a strained capsule and a smooth structure of the incision. The overview of autopsy characterized the acute toxicosis.

The microstructure of the liver of mice of this group was described the poorly distinguishable lobulation of the organ. The analysis showed the plethora of sinusoidal capillaries and the central vein, erythrocyte exudation behind the vascular walls that was typical indicator of erythrodiapedes. The research had seen the expansion of perisinusoidal spaces (Disse spaces). Centrolobular hepatocytes contained grit in the cytoplasm, in some of which the karyopicnosis was observed (Fig. 1). The activation of reticuloendotheliocytes with the increase in the number in the centrolobular sections of the lobules was considered in the research.

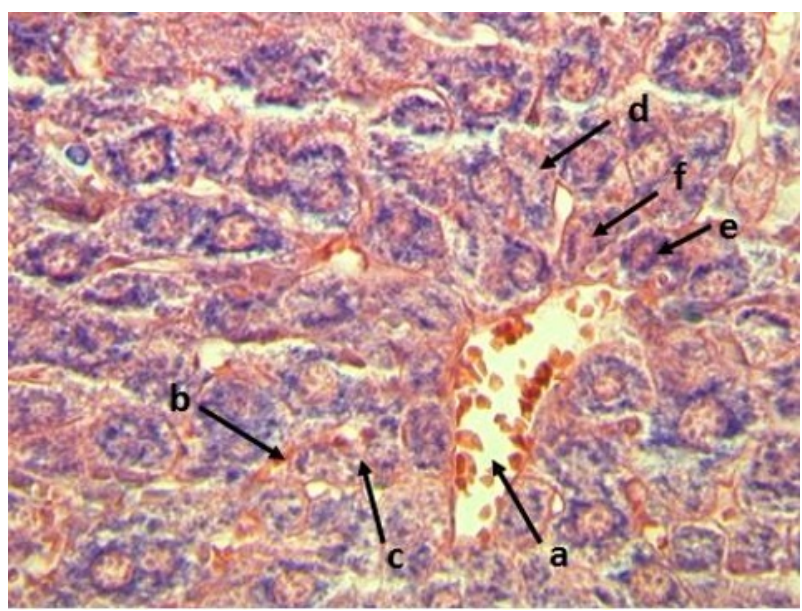

Fig. 1. The plethora of the central vein (a), sinusoidal capillaries (b), erythrodiapedes (c), granular dystrophy, karyopyknosis and karyolysis of hepatocytes $(\mathrm{g})$ of the mouse liver at a lethal dose $(3.0 \mathrm{~g} / \mathrm{kg})$ of nanostructured sapropel, stained with hematoxylin and eosin, X600

The autopsy of mice who received a toxic dose of nanostructural sapropel showed that it resulted in the erythrodiapedesis of the gastrointestinal mucosa. The study also noted the abundance of mucus in the stomach and the presence of clumps of sapropel in the mucous mass of the intestinal lumen. The kidneys, the spleen, and the liver were characterized by parenchymal dystrophy: volume increase, sagging consistency, and turbidity of the structure in the incision.

Histological analysis of the liver demonstrated the preservation of the girder-shaped structure, moderate plethora of the sinusoidal capillaries and the central vein followed by the expansion of the perisinusoidal spaces (Fig. 2). The centrolobular hepatocytes were characterized by deformation, some of which had destructive changes accompanied by the development of karyopiknosis, karyorhexis and karyolysis. In addition, the studies revealed the activation of reticuloendotheliocytes. Structural and functional changes of the liver corresponded to the development of toxic dystrophy of the organ.

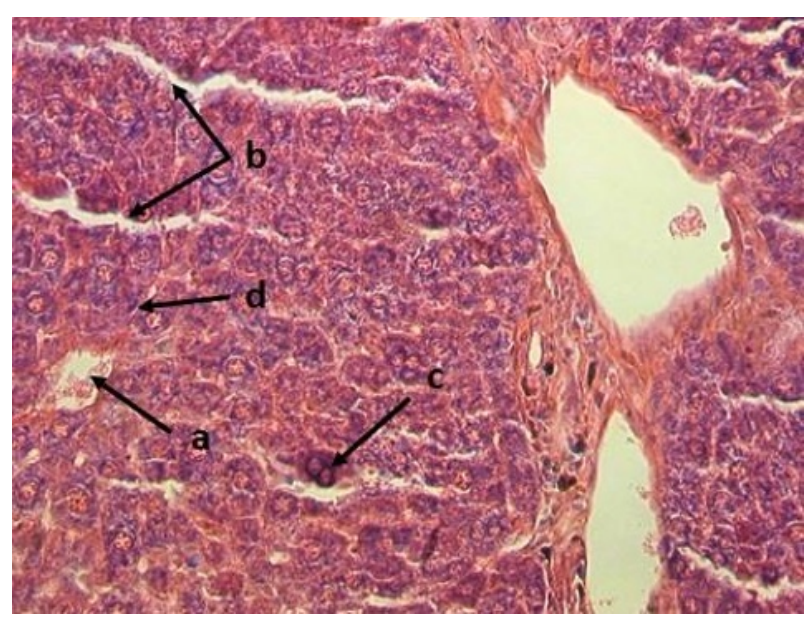

Fig. 2. Moderate plethora of central vein (a), sinusoidal capillaries (b), hepatocyte necrobiosis (c), reticuloendothelial cell activation (d) of the mouse liver at a toxic dose $(1.8 \mathrm{~g} / \mathrm{kg})$ of nanostructured sapropel, stained with hematoxylin and eosin, X400 
The internal organs of mice that received a safe dose of nanostructured sapropel, macroscopically visually did not have pathological changes. In the digestive tube we noted the preservation of the integrity of the mucosa, a moderate content of mucus and single small clumps of sapropel in the lumen. The volume, color, texture and shape of parenchymal organs (kidney, spleen, liver) corresponded to the age and species morphology criteria for this species of animals.

Histological examination of the liver pointed the retention of the beam structure of the organ (Fig. 3). The structures of the liver triad remained the same. The perivascular spaces of sinusoids were moderately expanded. The centrolobular and periportal hepatocytes had a polygonal shape with the preserving the structure of the cytoplasm and nucleus. Polyploid hepatocytes were detected in the periportal section of the hepatic lobules.

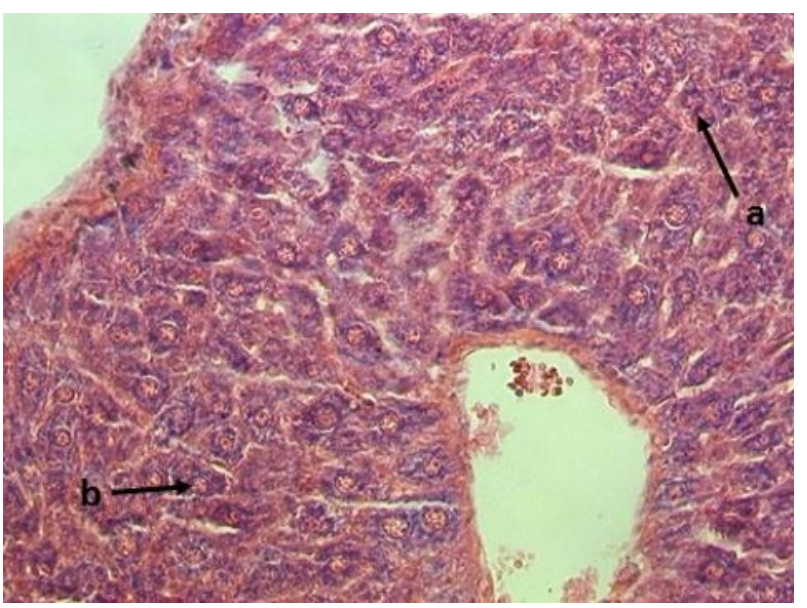

Fig. 3. The organization of hepatocytes into hepatic beams (a), polyploidy of periportal hepatocytes (b), severity of cytology of hepatocytes (c) of the mouse liver at a safe dose $(0.6 \mathrm{~g} / \mathrm{kg})$ of nanostructured sapropel, stained with hematoxylin and eosin, X600

\section{Discussion}

The choice of the liver as the organ that had no contact with the nanostructured sapropel for analyzing is justified by the fact that it is one of the main target organs and has various functions for maintaining homeostasis in a living organism.

The studies of many authors showed the change in the morphology of the organs of the gastrointestinal tract by direct contact of the nanoparticles with the surface of the organs. Nanostructured sapropel administered intragastrically in a lethal dose $(3.0 \mathrm{~g} / \mathrm{kg})$ caused destructive and necrotic changes in organs. Its administration in toxic doses $(1.2-2.4 \mathrm{~g} / \mathrm{kg})$ promoted the manifestation of focal dystrophy and deformation of cells of the mucous structures of the esophagus, stomach and intestines.

The introduction of safe doses $(0.3-0.6 \mathrm{~g} / \mathrm{kg})$ did not violate the integrity of the wall of the organs of the gastrointestinal tract and increased the secretion of mucus (Ezhkov et all., 2016).
The research determined the dose-dependent nature of the structural and functional state of the liver from the amount of introduced nanostructured sapropel. Nanostructured sapropel administered in a lethal dose due to the supply of a significant amount of chemically active nanoparticles caused the development of hemodynamic changes in the form of congestive hyperemia and erythrodiapedesis.

The expansion of Disse spaces could be considered a compensatory mechanism for reducing the concentration of nanoparticles. The great concentration of nanoparticles caused the granular dystrophy of the organ and destructive-necrotic lesions of hepatocytes largely in the centrolobular regions. The physiological response was an increase of the activity of reticuloendothelial cells in the central regions of the liver lobes.

Reducing the dose of nanostructured sapropel caused a decrease in the intensity of the manifestation of toxic action. In contrast to the lethal dose, moderate fullness of the blood vessels, focal necrobiosis and single necrosis of hepatocytes were observed during the research. An increase of the volume of reticuloendothelial cells in sinusoidal capillaries indicated the development of detoxication processes with the absorption and utilization of the amount of nanoparticles.

A safe dose of nanostructured sapropel contributed to the formation of polyploid cells in the periportal segments of the liver lobules. Polyploidy testified to the enhancement of the organ's reparative function. Activation of reticuloendothelial cells in combination with the formation of multicore hepatocytes could be considered as physiological protection with increased resistance.

\section{Conclusion}

The intragastric administration of nanostructured sapropel in the lethal, toxic and safe doses had a different effect on the morphology of the liver noncontact with nanoparticles organ. The features of the morphofunctional characteristics of the liver were established depending on the dose of the nanostructured sapropel.

The lethal dose caused hemodynamic disorders of the vessels (hyperemia, erythrodiapedes), focal necrosis and necrobiosis, granular dystrophy of hepatocytes.

The administration of the toxic dose of nanostructured sapropel leaded to moderate central venous and sinusoidal capillaries, necrobiosis of hepatocytes and activation of reticuloendothelial cells.

The safe dose injection of nanostructured sapropel noted the two- and multi-core periportal hepatocytes and moderate activation of reticuloendothelial cells.

\section{References}

1. N. Al-Beitawi, M. Shaker, K. El-Shuraydeh, J. Blaha, J. of applied animal res., 45, 543-549 (2017)

2. C. Buzea, I. Pacheco, K. Robbie, Biointerphases, 2, MR17-MR71 (2007) 
3. C. Constantinescu, R. Rizac, M. Militaru, Revista Romana de med. veter., 28, 13-21 (2018)

4. K. Elshuraydeh, N. Al-Beitawi, M. Al-Faqieh, Nanotechnol. Eng. Med., 5, 1-4 (2014)

5. V. Ezhkov, A. Ezhkova, A. Yapparov, I. Yapparov, I. Nizameev, E. Nefedyev, Nanotechnol. in Russia, 12, 438-443 (2017)

6. V. Ezhkov, A. Yapparov, A. Ezhkova, I. Yapparov, G. Ezhkova, R. Faizrakhmanov, T. Motina, Nanotechnol. in Russia, 11, 497-505 (2016)

7. R. Faizrakhmanov, Sci. notes of the Kazan State Acad. of Veter. Med. named after N.E. Bauman, 202, 199-202 (2010)

8. M. Gopi, B. Pearlin, R. Kumar, M. Shanmathy, G. Prabakar, Int. J. of pharmacol., 13, 724-731 (2017)

9. C. Hidayat, I. Sumiati, E. Wina, A. Jayanegara, Wartazoa-Buletin Ilmu Peternakan Dan Kesehatan Hewan Indonesia, 28, 107-118 (2018)

10. N. Higashi, N. Kojima, M. Miura, K. Imai, M. Sato, H. Senoo, Cell Tissue Res., 317, 35-43 (2004)

11. D. Jo, J. Bae, S. Chae, J. Kim, J. Han, D. Hwang, S. Lee, J. Kim, Mol. Cell. Proteomics, 15, 1681-1691 (2016)

12. D. Jo, J. Kim, T. Lee, J. Kim, Nanomed.nanotechnol. Boil. and med., 11, 1603-1611 (2015)

13. M. Nascarella, E. Calabrese, Dose-response, 10, 344-354 (2012)

14. A. Scott, K. Vadalasetty, A. Chwalibog, E. Sawosz, Nanotechnol. Reviews, 7, 69-93 (2018)
15. G. Sharma, V. Kodali, M. Gaffrey, W. Wang, K. Minard, N. Karin, J. Teeguarden, B. Thrall, Nanotoxicol., 8, 663-675 (2014)

16. V. Shkurupii, V. Gavrilin, G. Kovrigina, Ultrastructural pathology of the liver (Riga, 1984)

17. J. Siegel, K. Zaruba, V. Svorcik, K. Kroumanova, L. Burketova, J. Martinec, Nanoscale Res. Let., 13, 95-97 (2018)

18. J. Tang, M. Lu, Q. Fang, F. Lu, R. Shao, J. Shen, D. Lu, J. He, L. Lu, D. Niu, Animal product. Sci., 58, 1876-1884 (2018)

19. T. Terpinskaya, G. Zhavnerko, K. Yashin, V. Osipovich, E. Petrova, M. Artemyev, V. Ulashchyk, Nanotechnol. in Russia, 10, 303-310 (2015)

20. M. Tsoli, H. Kuhn, W. Brandau, H. Esche, G. Schmid, Small, 1, 841-844 (2005)

21. J. Wang, G. Zhou, C. Chen, H. Yu, T. Wang, M. Yongmei, G. Jia, Y. Gao, B. Li, J. Sun, Y. Li, F. Jiao, Y. Zhao, Z. Chai, Toxicol. Letters, 168, 176-185 (2007)

22. M. Ware, B. Godin, N. Singh, R. Majithia, S. Shamsudeen, R. Serda, K. Meissner, P. Rees, H. Summers, ACS NANO, 8, 6693-6700 (2014)

23. D. Warheit, K. Reed, T. Weeb, Exp. Lung Res., 29, 593-606 (2003)

24. Y. Zhang, D. Xu, W. Li, J. Yu, Y. Chen, J. of Nanomater., 375496 (2012) 